\title{
Synote Usage on E-learning Search
}

\author{
Kavitha Chandrakanth , Latha Parthiban
}

\begin{abstract}
E-Learning plays a necessary role in the way of searching a secured educational video resources for the students. In this paper, e-learning domain introduces a concept of searching a video resources in annotated way with linked data cloud. The purpose for the student to choose e-learning is that the learner can continue learning anywhere and at any time. This paper goes with unique approach of synote tool where synote tool allows the e-learners to search the syntactic web information accurately and with different online video resources. Linked data cloud concept is applied in this concept to search a secured search. The result is proved for secured search of educational video resources and shows the reusability of resources for $e$-learners.
\end{abstract}

Keywords :E-learning, educational video resources, linked data cloud, synote, syntactic web information.

\section{INTRODUCTION}

Education has been given a important role to lead a best carrier in life. E-learning is mostly preferred by the student who wants to learn more at any time and at any place Facebook ,Twitter and You tube are some of the social media provide a better opportunities for the student of E-learners .Student can withstand with more knowledge and power learning in social media. ,

A research survey of traditional way of learning needs a class room ,instructor are been centered, time and location are to be fixed and the material cannot be reused. Pedagogy is the traditional way of obtaining knowledge in the fixed environment like class room and with the material usage of chalk, black board ,etc.[1\},Another survey of M-learning refer specifically via mobile devices ,learning on the go and allows the learning outside the class room. In M-learning the disadvantage is educational video resources cannot be reused by the students[2].E-learning domain has a better scope for students in search of video resources with quality and quantity search.

The proposed E-learning has several advantages as follows :

1.Relatively established concept of learning.

2.Cost effective for students.

3.Provides unlimited access of knowledge .

.4.Allows the student to learn outside the

Revised Manuscript Received on December 30, 2019.

* Correspondence Author

Kavitha Chandrakanth, Research Scholar, Department of Computer Science and Engineering, Sathyabama Institute of Science and Technology, OMR Road, Chennai. Email: kavitha.ck13@gmail.com

Latha Parthiban*, Department of Computer Science, Pondicherry University CCy, Lawspet, Pondicherry. Email: lathaparthiban@yahoo.com

(C) The Authors. Published by Blue Eyes Intelligence Engineering and Sciences Publication (BEIESP). This is an open access article under the CC BY-NC-ND license (http://creativecommons.org/licenses/by-nc-nd/4.0/)
Classroom.

5.Reusability and sharing of educational video resources. The annotation is done by synote tool. Annotation are been categorized into four categories for the student to search an efficient video resources.

1.Source Creators :The person who are well known about the resources.

2.Syllabus framers : The people who are commanders to frame the resource $s$ syllabus according to the usage of learners.

3.Guiders :The person who give lecturer with clear description of resource.

4 Students :The learner who undergo elearning courses.

Annotation concept collage with Linked Open Data cloud to obtain the educational video resources from the source creators .Linked Open data with synote tools makes the syllabus framers to frame the syllabus accordingly and upload into the online platform for guiders and students [5]. Synote tool periodically provides a secured search of video resources for guiders and students. Reusability of resources are obtained by ranking system. The resources usage often and voting rates of the students shows the reusability.

\section{PROPOSED ARCHITECTURE}

The proposed architecture is divided into four sectors course creators, syllabus framers, guiders and students as shown in Fig.1.The course creators create the the educational video resources with the URL in link data cloud data and store the information of resources .Course creators are called as experts who are allowed to edit the video resources such as adding a particular video or deleting a particular video resources according to the need of E-learners. The resources are then stored in data base .Framers frame the syllabus according to the curriculum for guiders to deliver the notes to the students The framers checks on semantic web and Passes their work to guiders. Guiders delivers the framed syllabus in any format such as class room method, by power point or in note book format. Data are annotated by synote tool [6]. 


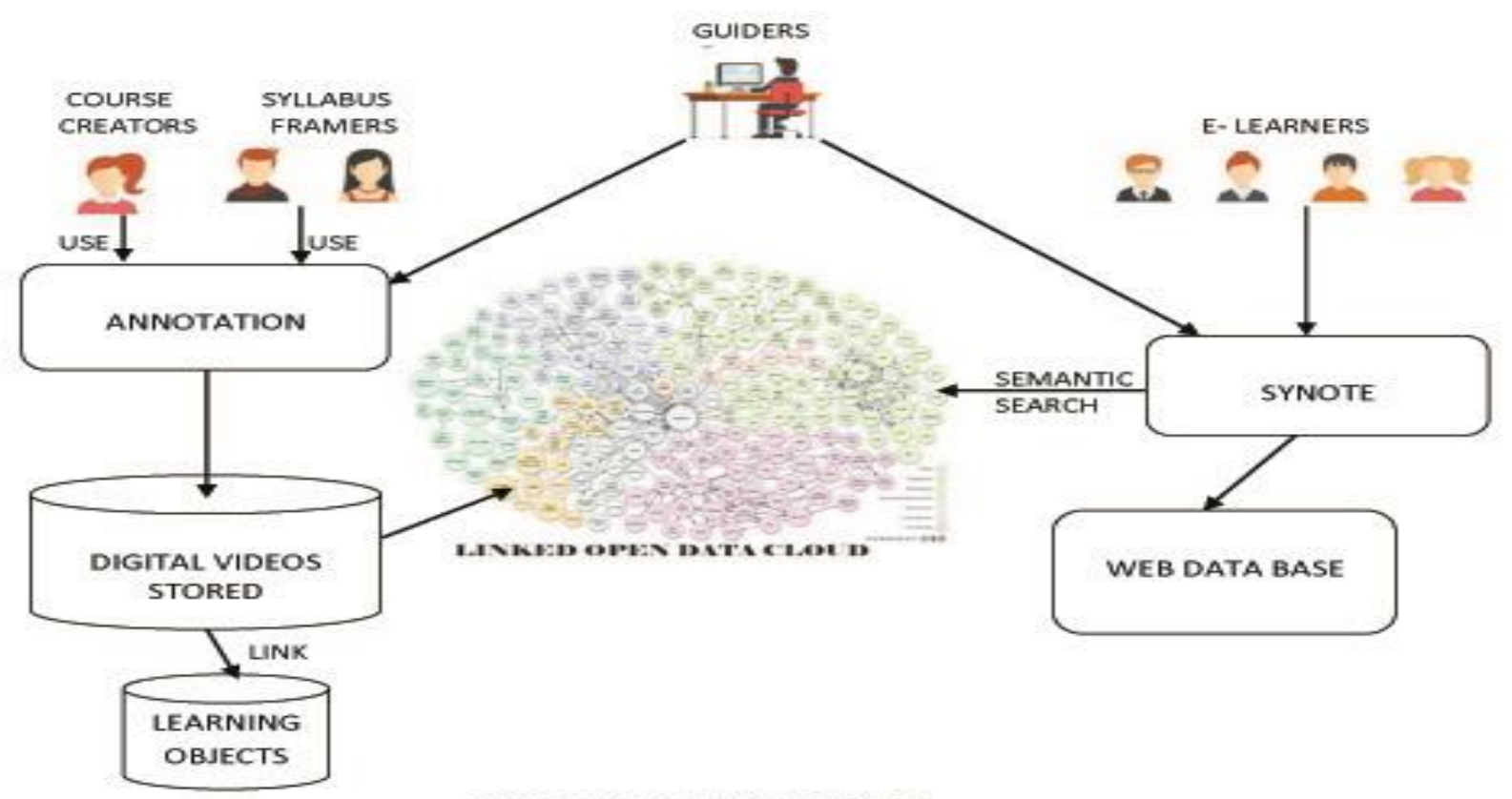

OVERALL ARCHITECTURE

Fig.1 Proposed Architecture

The use of synote tool helps to retrieve data faster and the students can gain intellectual knowledge with online browsing platform .Semantic search with linked open cloud are available for search from semantic web.[3].

\section{A. Annotation tool}

Annotation ontology includes several processes:

1.Friend of a Friend :Find out a user from URI's [4].

2.Duration Ontology : Finds a certain user for certain time period .

Linked open data with annotation ontology technology gives information on the use of free spell of grammar and storage requirement of digital library [7].

A sample pseudo code working of annotation tool is as follows :

\section{/* Encrypt */}

$<$ head $>$

$<$ title $>$ e-learning $</$ title $>$

$<$ script type="text/javascript" >

$\$$ (document).ready(function()

\{

\$("\#btnUpdate").click(function(event)

\{

var password = \$("\#password").val();

if (password != ")

\{

document.PasswordAction.action=

'PasswordAction.action';

document.PasswordAction.submit();

\} else

\{

alert('Please enter the password !!');

\}

\});

\}$)$;

$<$ script $>$

/* Upload */

$<$ head $>$

$\$$ (document).ready(function()

\{

\$("\#btnUpdate").click(function(event)

\{

var tag $=\$(" \#$ tag").val();

if(tag!=")

\{

document.uploadAction.action=

'FileUploadAction.action';

document.uploadAction.submit();

\}

else

\{

alert('Please enter the tag field !')

\}

\});

\}$)$;

$</$ script $>$ 


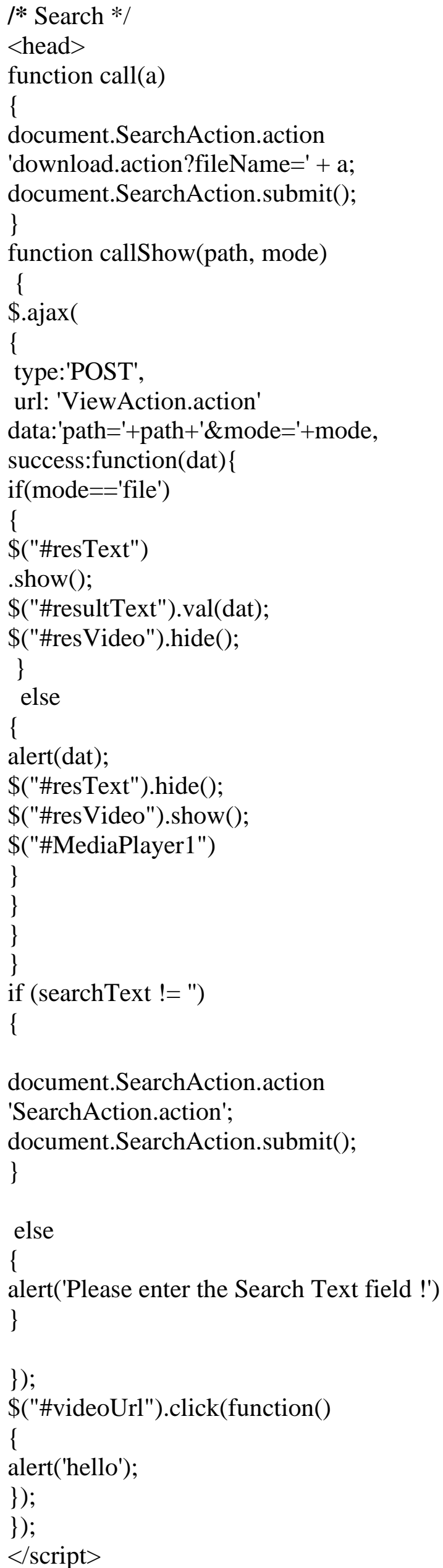

\section{B. Synote technology working}

Many methods of traditional annotation of videos uses a free keyword search to retrieve a semantic resources but they fails

$=$ in multilingual performance coverage of educational video resources [8][9]. The video resources muat be accurate and it should be of machine understandable. An integrated terminology will able to provide the machine understandable form..Synote provides the accuracy for retrieving the completion of video resources.

A sample Pseudo code for video play retrieval of resources

$<\mathrm{html}>$

$<$ head $>$

$<$ script $>$

function callme()

\{

ShutMeDown();

MediaPlayer1.FileName="1.avi";

StartMeUp();

\}

$</$ script $>$

$</$ head $>$

$<$ body background="'" text="red" $>$

$<$ object id="MediaPlayer1" width="350" height="350"

classid="CLSID:22D6F312-B0F6-11D0-94AB-0 080C74C7E95"

codebase="http://activex.microsoft.com/activex/c ontrols/mplayer/en/nsmp2inf.cab\#Version=5,1,52, 701"

standby="Loading Microsoft ${ }^{\circledR}$ Windows ${ }^{\circledR}$ Media Player components..."

type="application/x-oleobject" align="middle">

$<$ param name="FileName" value="1.avi" $>$

$=\langle$ paramname $=$ "ShowStatusBar" value $=$ "True" $>$

<paramname="DefaultFrame"

value $=$ "mainFrame" $>$

$<$ param name="autostart" value $=$ "true" $>$

$<$ embed type="application/x-mplayer2"

pluginspage $=$

http://www.microsoft.com/Windows/MediaPlayer /"

$\mathrm{src}=" 1 . a v i "$

autostart="true"

align="middle"

width="176"

height="144"

defaultframe="rightFrame"

showstatusbar="true">

$<$ embed $>$ 


\section{Synote Usage on E-learning Search}

\author{
$<$ object $>$ \\ $<$ form $>$ \\ $<$ input type=button name $=b$ value $="$ Click" \\ onclick="callme()" $>$ \\ $</$ form $>$ \\ $</$ body $>$ \\ $</$ html $>$
}

\section{EXPERIMENTAL RESULTS}

The evaluation process of synote tool fulfills the needs of students. knowledge gainer criteria are satisfied as follows :

1.Link open data cloud finds a new URI's.

2.Annotated way of search finds new content on demand of the E-Learners.

3. Related information of resources either video or text based search are satisfied by the need of user [10].

4.Knowledge gainer can search the content actively by anytime and any where.

A survey was done for 100 students for E -Learning students as shown in Fig .2.It identifies the learners to retrieve relevant information using semantic web within a time limit of 5 minutes ,10 minutes or 15 minutes.

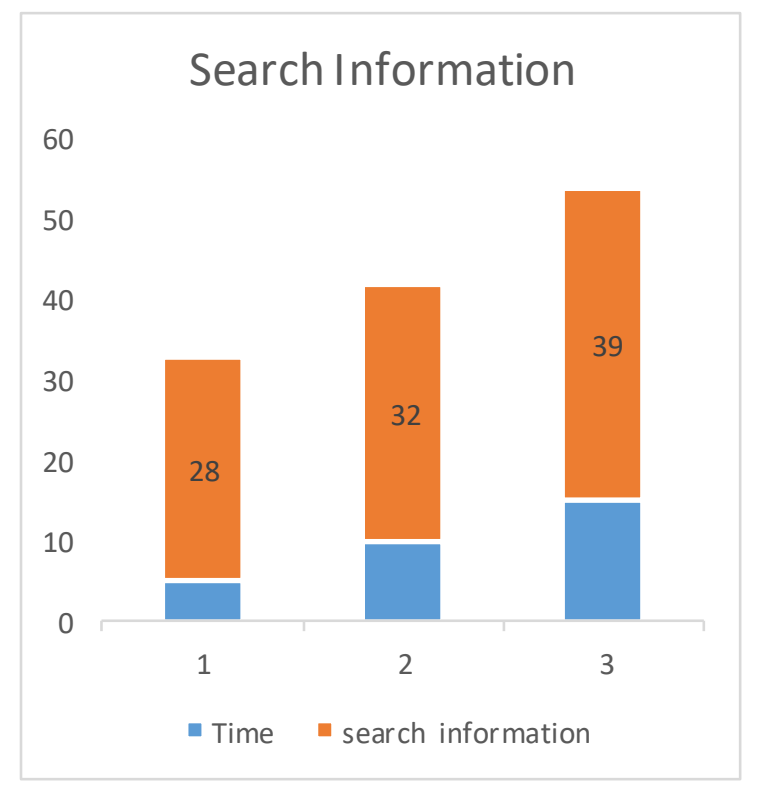

Fig.2 Search Information

The E-Learners were allowed to retrieve the information using Linked open cloud on the annotated way on the topic " Methods used to save water in houses ".The content search by knowledge gainer was searched for 5 minutes ,then allowed to searched for 10 minutes and finally for 15 minutes. The result of seaarched information is as 28 ,32 and 39 respectively as shown in Fig.2. Before the searching process getting started the E-learners has to get registered and also the course creators and and syllabus framers has to get registered as shown in Fig.3.

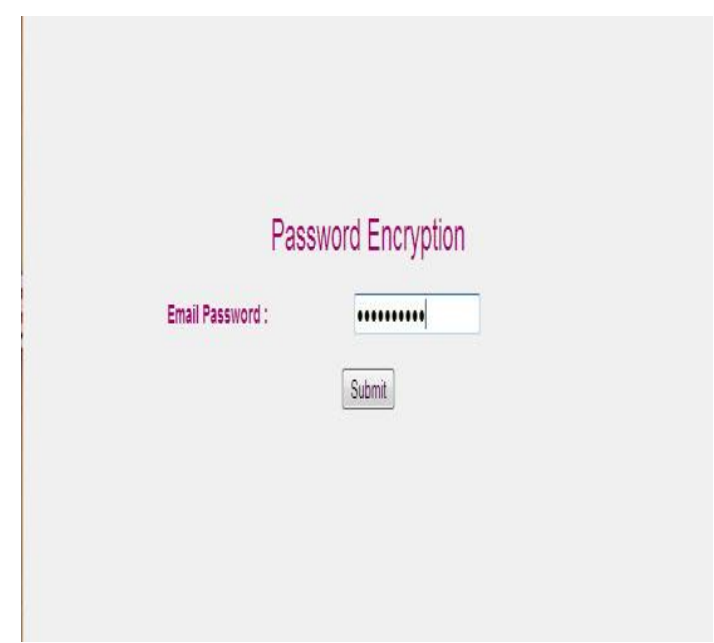

Fig.3 Registered Learners

When the learners got registered the forms the materials can be viewed by the learners and the help can be obtained from the guiders and the E-Learners can gain knowledge at any time Semantic search done by synote technology is shown in Fig.4

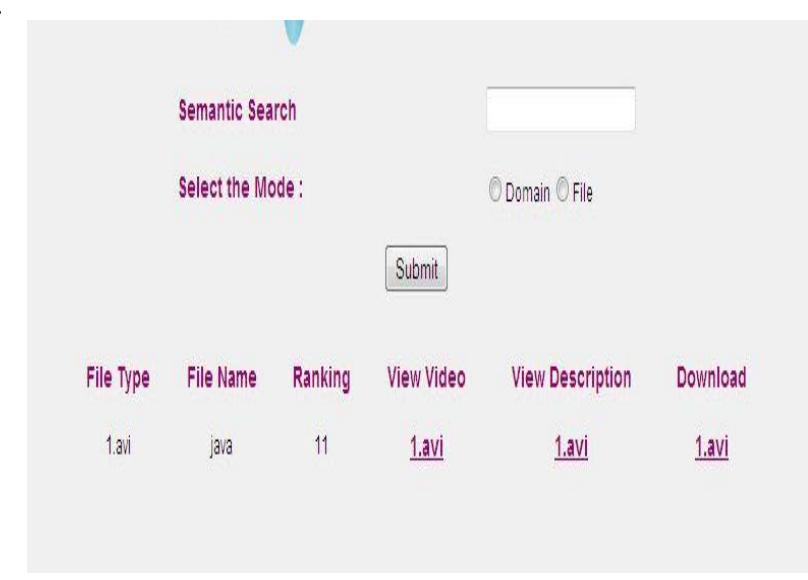

Fig.4 Synote search

The way of E-learners teaching and usage of learning plays a major role of the web. Billions of video resources are available and the annotation part becomes simple to download. The result shows the usage of E-Learners which is in Fig.5 


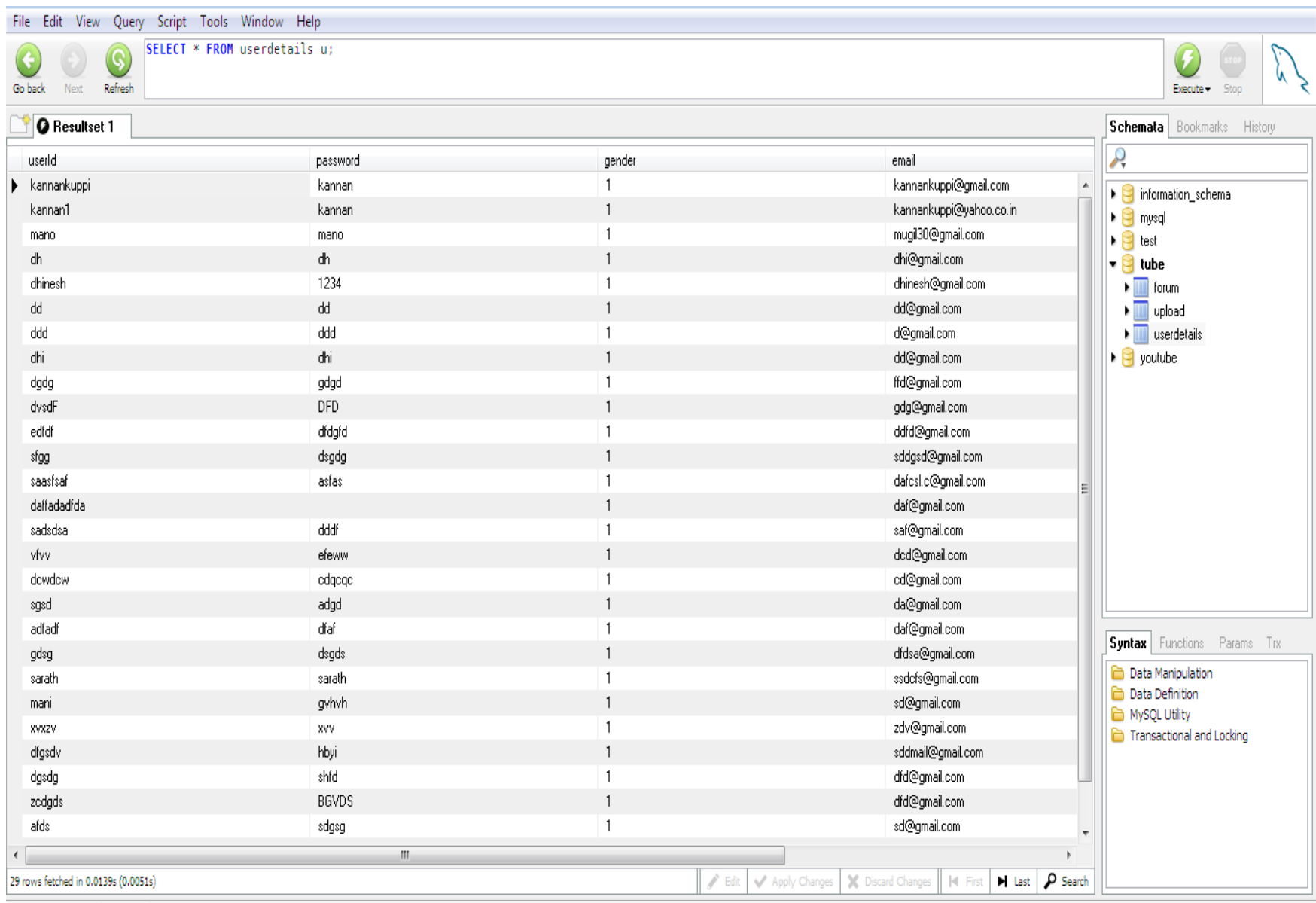

Fig. 5 Usage of E-Learners

\section{CONCLUSIONS}

This work does synote search in an efficient way with the linked open data cloud in the annotated web .The paper concludes the followings:

1.Optimal Search on usage of synote with the various available of video resources .

2.The semantic web search proves the spell free of grammar.

3.Easier and quick way of learning.

4.Faster search by synote.

\section{REFERENCES}

1. .Xin Chen ,Michaela Voprvoreanu and Krishna Madhavan ,'Mining Social Media Data For Understanding Student's klearning Experiences “ ,IEEE Transaction on Learning Technologies ,Vol. 7,No.3 ,July -September 2014.

2. S. Wei, Y. Zhao, Z. Zhu, and N. Liu, "Multimodal Fusion for Video Search Reranking," IEEE Trans. Knowledge and Data Eng.,vol. 22, no. 8, pp. 1191-1199, Aug. 2010.

3. M. Hausenblas and M. Karnstedt, "Understanding Linked Open Data as a Web-Scale Database," Proc. Second Int'l Conf. Advances in Databases Knowledge and Data Applications (DBKDA), pp. 56-61, Apr. 2010.

4. Hong Qing Yu,CarlosPedrinaci, Stefan Dietze ,and John Domingue "Using Linked Data to and Annotate and Search Educational Video Resources for Supporting Distance learning",IEEETrans.LearningTechnologies.,vol.5,no.2, April 2012.

5. J. Broekstra, A. Kampman, and F.V. Harmelen, "Sesame: A Generic Architecture for Storing and Querying RDF and RDF Schema," Proc. First Int'l Semantic Web Conf. the Semantic Web, pp. 54-68, 2002.

6. D.Brickley and L.Miller,'FOAF Vocabulaty Specification 0.921,’http://xmlns .com/foaf/spec,2007.

7. T.Berners-Lee, J.Hendler ,andO.Lassila , "The Semantic Web ,' Scientific Am. MagaZine ,2001.
8. L. Ballan, M. Bertini, A.D. Bimbo, and G. Serra, "Video Annotation and Retrieval Using Ontologies and Rule Learning," IEEE Multimedia, vol. 17, no. 3, pp. 72-76, Oct.-Dec. 2010.

9. Brickley, D. Basic Geo (WGS84 lat/long) Vocabulary (2004) Online at http://www. W3.

10. K.Vijayakumar ,C.Arun ,"Automated risk identification using NLP in cloud based development environment Ambient Intell Human Computing “, Springer DOI 10.1007/12652-017-0503-7 May 2017.

\section{AUTHORS PROFILE}

Kavitha Chandrakanth is pursuing her $\mathrm{PhD}$ in CSE from Sathyabama University and is in teaching in Engineering college for past 10 years. She has published 2 scopus indexed journals and presented papers in 2 international conference.

Latha Parthiban studied her BE from Madras University, ME from Anna University and $\mathrm{PhD}$ from Pondicherry University. She has over 150 peer reviewed journals and has guided $18 \mathrm{PhD}$ scholars. She is a life memner of CSI,ISTE and many professional societies like IEEE. 